EPJ Web of Conferences 53, 09005 (2013)

DOI: $10.1051 /$ epjconf/20135309005

(C) Owned by the authors, published by EDP Sciences, 2013

\title{
Calibration and testing of a prototype of the JEM-EUSO telescope on Telescope Array site
}

\author{
M. Casolino, a for the JEM-EUSO collaboration ${ }^{\mathrm{b}}$, T. Fujii $^{2}, \mathrm{D}^{1}$ Ikeda $^{3}$, \\ Y. Tameda ${ }^{3}$, T. Shibata ${ }^{3}$, H. Sagawa ${ }^{3}$, M. Fukushima ${ }^{3}$, J.N. Matthews ${ }^{4}$, \\ G.B. Thomson ${ }^{4}$, M. Takeda ${ }^{3}$, S. Ogio ${ }^{2}$, Y. Tsunesada ${ }^{5}$, T. Tomida ${ }^{1}$, J. Belz $^{4}$ \\ and P. Sokolsky ${ }^{4}$
}

${ }^{1}$ RIKEN, ASI Institute and INFN Sezione di Roma Tor Vergata

${ }^{2}$ Osaka City University Faculty Administration Department, Sugimoto-ku, Osaka 558-8585, Japan

${ }^{3}$ Institute for Cosmic Ray Research, University of Tokyo, Kashiwa, Chiba 277-8582, Japan

${ }^{4}$ Institute for High Energy Astrophysics and Department of Physics, University of Utah, Salt Lake City, UT 84112-0830, USA

${ }^{5}$ Graduate School of Science and Engineering, Tokyo Institute of Technology, Meguro, Tokyo 152-8551, Japan

6 Interdisciplinary Graduate School of Medicine and Engineering, Mechanical Systems Engineering, University of Yamanashi, Kofu, Yamanashi 400-8511, Japan

\begin{abstract}
Aim of the TA-EUSO project is to install a prototype of the JEM-EUSO telescope on the Telescope Array site in Black Rock Mesa, Utah and perform observation of natural and artificial ultraviolet light. The detector consists of one Photo Detector Module (PDM), identical to the 137 present on the JEMEUSO focal surface. Each PDM is composed by 36 Hamamatsu multi-anode photomultipliers (64 channels per tube), for a total of 2304 channels. Front-End readout is performed by 36 ASICS, with trigger and readout tasks performed by two FPGA boards that send the data to a CPU and storage system. Two, 1 meter side square Fresnel lenses provide a field-of-view of 8 degrees. The telescope will be housed in a container located in front of the fluorescence detector of the Telescope Array collaboration, looking in the direction of the ELF (Electron Light Source) and CLF (Central Laser Facility). Aim of the project is to calibrate the response function of the EUSO telescope with the TA fluorescence detector in presence of a shower of known intensity and distribution. An initial run of about six months starting from end 2012 is foreseen, during which we expect to observe, triggered by TA electronics, a few cosmic ray events which will be used to further refine the calibration of the EUSO-Ground with TA. Medium term plans include the increase of the number of PDM and therefore the field of view.
\end{abstract}

\section{INTRODUCTION}

The Extreme Universe Space Observatory on the Japanese Experiment Module (JEM-EUSO) of the International Space Station (ISS) is the first mission that will study Ultra High-Energy Cosmic Rays (UHECR) from space [4, 8]. JEM-EUSO will observe Extensive Air Showers (EAS) produced by UHECRs traversing the Earth's atmosphere from above. For each event, the detector will make accurate measurements of the energy, arrival direction and nature of the primary particle using a target volume

\footnotetext{
a e-mail: casolino@roma2.infn.it

bFor the full authorlist see Appendix "Collaborations" in this volume

This is an Open Access article distributed under the terms of the Creative Commons Attribution License 2.0, which permits unrestricted use, distribution, and reproduction in any medium, provided the original work is properly cited.
} 


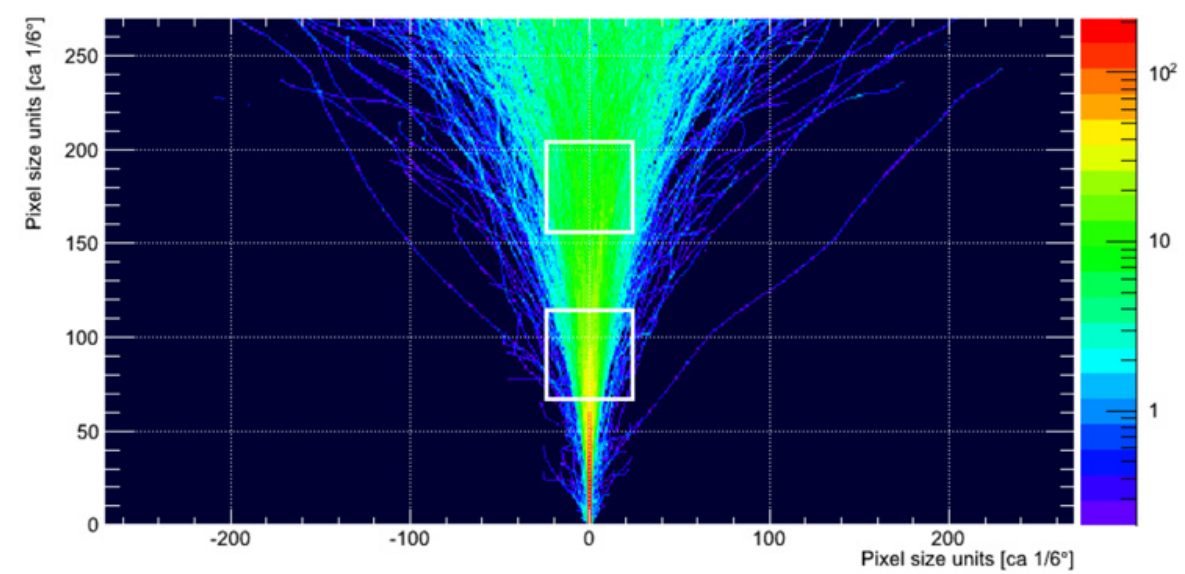

Figure 1. Simulation of the UV photons generated by the TA ELS, Electron Light Source, as seen from the EUSOGround detector. The white square shows the field-of-view of the EUSO-Ground telescope.

far greater than what is achievable from ground. The corresponding increase in statistics will help to clarify the origin and sources of UHECRs as well as the environment traversed during production and propagation. Possibly, this will bring new light onto particle physics mechanisms operating at energies well beyond those achievable by man-made accelerators. The spectrum of scientific goals of the JEM-EUSO mission includes as exploratory objectives the detection of high-energy gamma rays and neutrinos, the study of cosmic magnetic fields, and tests of relativity and quantum gravity effects at extreme energies. In parallel JEM-EUSO will systematically perform observation of the surface of the Earth in the infra-red and ultra-violet ranges, studying also atmospheric phenomena (Transient Luminous Effects). The apparatus is a 2 ton detector using Fresnel-based optics to focus the ultraviolet (UV) light from EAS on a focal surface composed of about 6,000 multianode photomultipliers for a total of $\simeq 3 \cdot 10^{5}$ channels. In the framework of the EUSO project, a number of prototype detectors are being realized to calibrate the detector response, test its performance in air and space, raise the Technological Readiness Level of some of the components, improve our knowledge of the various detectors. These projects include a series of stratospheric balloon flights (EUSO-BALLOON [1]) and a ground calibration with the Telescope Array collaboration (TA-EUSO).

\section{TA-EUSO}

The TA-EUSO project aims to install a fully functional prototype of JEM-EUSO on Black Rock Mesa, the site of one of the fluorescence light detector of the Telescope Array collaboration. From there it will observe artificial light (Laser and electron-generated UV) and events coming from cosmic rays.

Aim of the project is to calibrate the detector with UV light coming from known sources such as the ELS - Electron Light Source, and laser from the CLF (Central Laser facility). Furthermore the response to artificial light will be correlated with that of the TA Fluorescence light telescope in order to calibrate the EUSO-Ground response with that of TA and reduce the systematic of the measurement.

Also UV light coming from cosmic ray events will be detected by EUSO-Ground with an external trigger coming from TA. In this case studies of the tranversal profile of the shower will be performed. Note that in the first stage of the project, the use of one PDM will only allow to see part of the shower (Fig. 1), albeit with an higher spatial resolution than TA Fluorescence detector. In subsequent stages it will be considered the addition of other PDM detectors (with the same optics) to enlarge the field of view. 


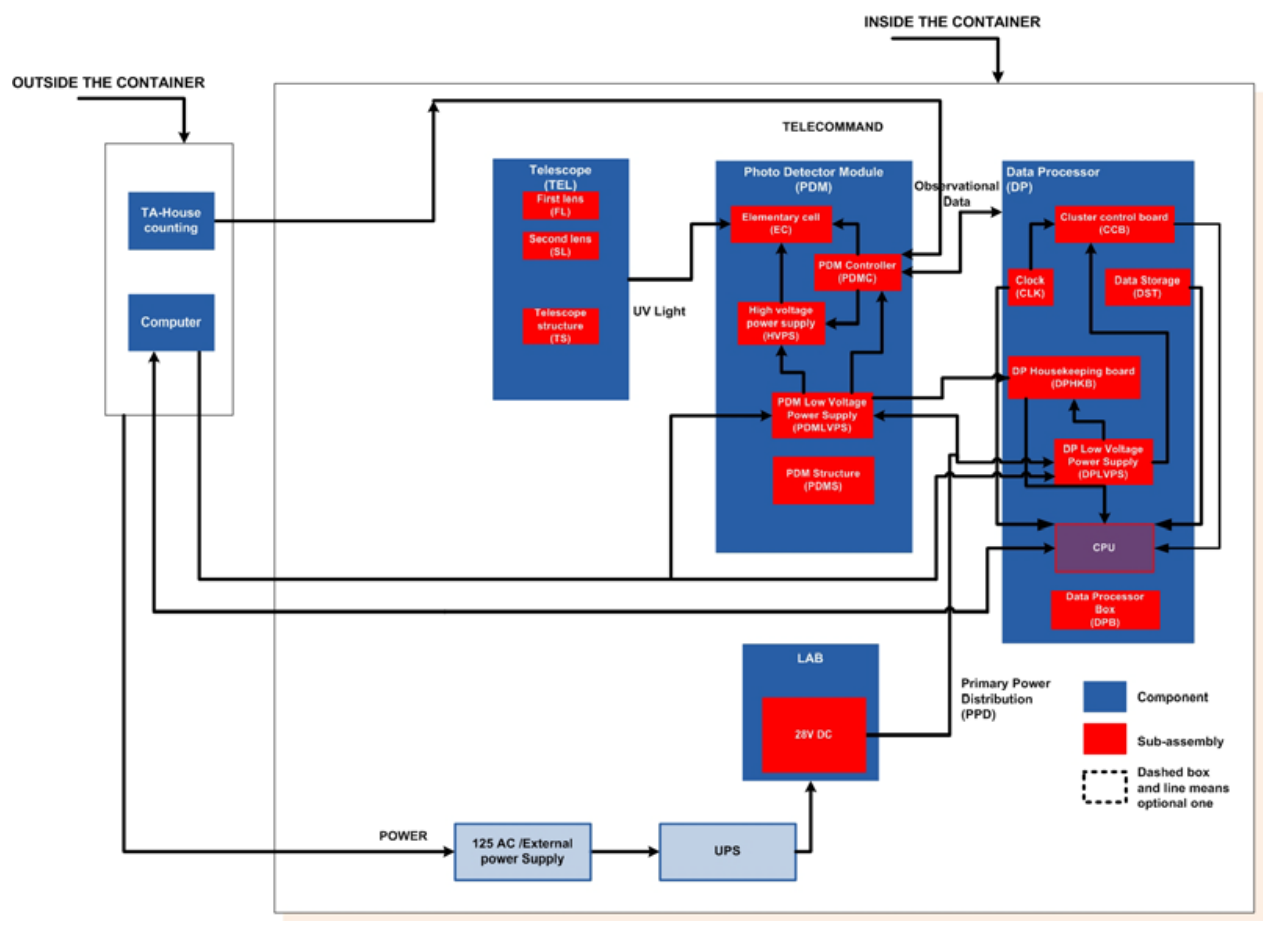

Figure 2. Block Scheme of EUSO-Ground detector, with the subsystems and their relationship.
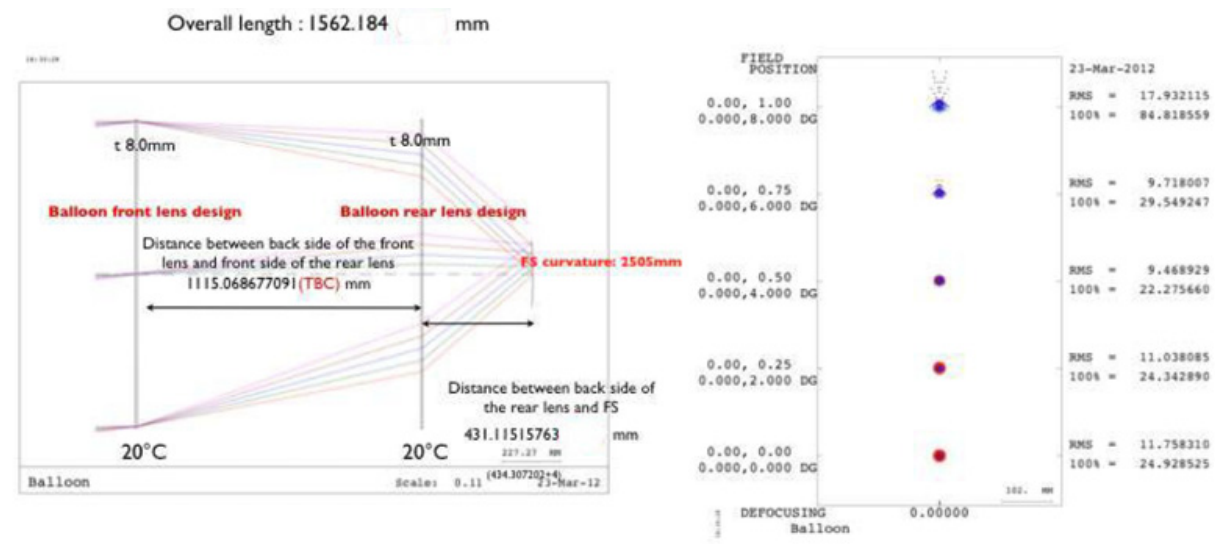

Figure 3. Optics design of the EUSO-Ground detector. Two Fresnel lenses of $1 \mathrm{~m}$ side (left and center) focus the signal on the PDM (right). Note that the lens design is the same of the balloon, in order to keep the two instruments as close as possible. Furthermore, the Focal surface curvature is the same of JEM-EUSO for the same reasons.

In Figure 2 is shown the functional block diagram of the telescope. It is housed in a container about $20 \mathrm{~m}$ in front of the TA Fluorescence detector of Black Rock Mesa. As mentioned, in the telescope field of view lie both ELS and the CLF, so that light from these sources can be detected by the two double sided Fresnel lenses optical system. The optical signal is focused on and is detected by the PDM. The PDM is attached to the telescope (TEL) with alignment accuracy better than $0.1^{\circ}$. 


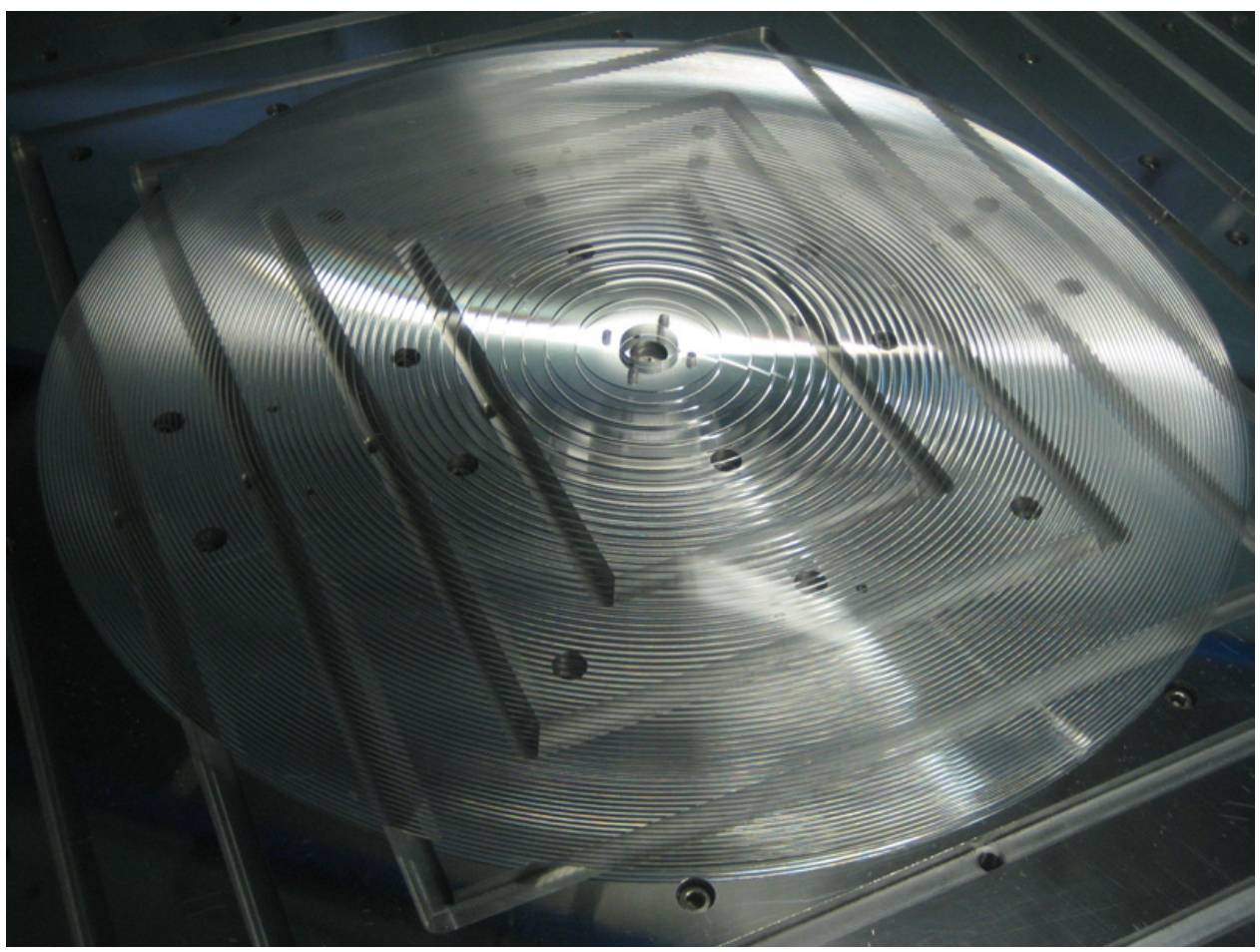

Figure 4. Picture of the rear Fresnel lens under manufacturing.

The detector consists of two square Fresnel lenses, 1 meter side $\left(4\right.$, focusing the light in a $\pm 4^{\circ}$ field of view on one PDM (Photo-Detector-Module) of 2304 pixels 3. Each PDM has a focal surface of 13.6. $13.6 \mathrm{~cm}^{2}$ and is composed by 36 Hamamatsu multi-anode photomultipliers (64 channels per tube), for a total of 2304 channels. They are arranged in a $6^{*} 6$ element array, with front-end readout performed by 36 ASICS (SPACIROC). Readout tasks are performed by an FPGA board that stores the data in a 100 GTU (Gate Time Unit. 1 GTU $=2.5 \mu s$ ) round buffer.

In case of an event (trigger, pedestal or calibration) data are sent to a second FPGA for further processing and interfacing with the CPU. Note that the electronic system and the $2.5 \mu \mathrm{s}$ sampling rate are designed for observation of UHECR showers from the altitude of $400 \mathrm{~km}$ of altitude of the Space Station. Therefore the relative proximity of cosmic ray events and the limited field-of-view seen from ground is such that the shower is visible only in one-two time frames (GTUs). Therefore, a dedicated trigger system for the ground detector will be realized. However-given the limited number of GTUs available-this is expected to have low efficiency: therefore an external trigger, generated from TA trigger electronics, will be used for the main cosmic ray acquisition.

\subsection{Data acquisition and reduction}

The Data acquisition system (Fig. 5) is based on an architecture capable of reducing at each level the amount of data through a series of triggers controlling an increasingly growing area of the focal surface $([2,5])$. On ground it is necessary to reduce the $1 \mathrm{Gbyte} / \mathrm{s}$ background output on the focal surface (FS) to 10-100 Gbyte/day which can be stored on the ground. Each board and data exchange protocol is therefore capable to handle the data and send them to the higher level of processing if they satisfy the trigger conditions. This structure is similar to that expected on board the International Space Station, where most of the triggers will be due to noise. 


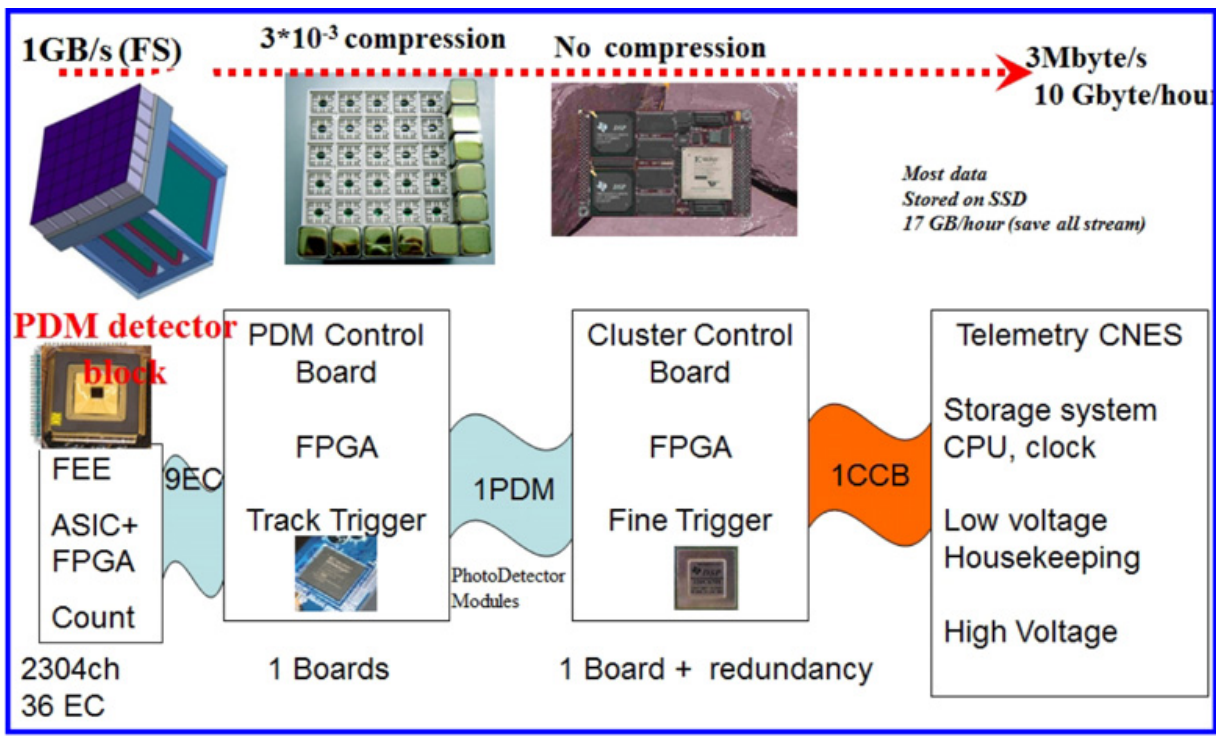

Figure 5. Data Reduction scheme. Each of the $\simeq 36$ Multi-Anode Photo Multiplier (MAPMT) of the focal surface is read by an ASIC digitizing the photoelectron signal. A $6 * 6$ array of MAPMT is present and read by an FPGA which performs first level triggering and rejects noise by three orders of magnitude. The general acquisition and data storage is performed by the main CPU (right).

An ASIC chip (SPACIROC, Spatial Photomultiplier Array Counting and Integrating Readout Chip) performs photo-electron signal readout and conversion for the 64 channels of the MAPMT. It has two main purposes: counting the number of photons reaching each pixel of the MAPMTs and measuring the intensity of photon flux by performing charge to time (Q-to-T) conversion. The first version of the ASIC is the result of the collaboration between OMEGA/LAL-Orsay, France, RIKEN, ISAS/JAXA and Konan University, Japan. It has 64 channels preamplifier with independent gain (8-bit) adjustment in order to correct for the non-uniformity of the 64 MAPMT anodes; photon counting for each channel with a system managing $100 \%$ trigger efficiency for a charge greater than $50 \mathrm{fC}(1 / 3$ p.e for a MAPMT gain of $10^{6}$ ) and a double pulse resolution as close as possible to $15 \mathrm{~ns}$.

Q-to-T converter with an input charge range of $2 \mathrm{pC}-200 \mathrm{pC}$ (12.5 p.e-1250 p.e.). The last dynode signal, produced by the MAPMT, has to be processed as well as 8 internal channels corresponding to the sum of up to 8 channels signals. The chip has also a low power consumption of about $1 \mathrm{~mW} / \mathrm{channel}$.

An FPGA (Xilinx XC6VLX240T) board handles first level trigger data on a PDM level (reading 36 MAPMTs). Background events are rejected by a factor $10^{3}$. Second level triggering algorithms is implemented by the CCB (Cluster Control Boards), DSPs with about 1Gflop computing capability which further process the data. At this level background is rejected by another factor $10^{3}$. The CPU has a relatively low processing power $(100 \mathrm{MHz})$ since it is in charge of the general handling of the experiment. The CPU is part of the Storage and Control Unit System (SCU), the evolution of a similar system used for PAMELA ([3]) and composed of a number of boards devoted to different tasks: 1. CPU main board 2. Mass Memory (8 Gbyte) 3. Internal and external housekeeping interfaces (CAN bus) 4 . Hard Disk storage Data acquisition and status of the apparatus can be monitored in remote from a PC in the counting room of TA building. All hardware, software reset, as well as power cycling, can be performed in remote in order to avoid access to the EUSO-Ground container during data acquisition in case of malfunction. Only high level data, coming from artificial light sources and cosmic ray events will be transferred via network, wehereas the calibration and pedestal raw data will be physically transferred to an higher (Grid-based) link location. 


\subsection{Slow control and Housekeeping}

The housekeeping module is connected to the CPU with the task to distribute commands to the various detectors and to collect telemetry for them in order to monitor in real-time the status of the experiment and optimize its observational parameters. The module is capable of handling both single (upon request) or cyclic (periodic) acquisition/commanding operating both is possible according to the acquisition program and status. Different acquisitions and controls are foreseen. For instance all relays to switch on / off secondary power supplies and subsystems are controlled by High Level signals. This approach has the advantage of a great degree of flexibility keeping at the same time a strong robustness and reliability.

\section{CONCLUSION}

JEM-EUSO aims to perform an high-statistic UHECR measurement from space. To achieve this goal a number of technologies and techniques will be used. In order to qualify them for space, raise the Technological Readiness Level, and optimize their performances, a number of laboratory tests using accelerator, thermal vacuum chambers are being carried out on the single elements. In parallel, several observation campaigns from ground and air-using scaled-down versions of the JEM-EUSO telescopewill perform full functional tests of the apparatus, optimize the observation techniques and improve the general performance of the apparatus. The realization of the JEM-EUSO telescope, now concluding phase A study, is consistent with, and will benefit from the time scale of these campaigns. Furthermore, also after flight of JEM-EUSO, a series of ground detectors such as EUSO-Ground on TA site, will help in reducing the systematic error between the various experiments.

\section{References}

[1] P. von Ballmoos et al.: The EUSO-BALLOON project, these proceedings

[2] Casolino, M.: The data acquisition and handling system of JEM-EUSO experiment, Nuclear Instruments and Methods in Physics Research Section A: Accelerators, Spectrometers, Detectors and Associated Equipment 623, 516-518, 1st International Conference on Technology and Instrumentation in Particle Physics, 2010

[3] Casolino, M. et al.: Advances in Space Research 37, 1857-1861, 2006

[4] Casolino, M. et al.: Detecting ultra-high energy cosmic rays from space with unprecedented acceptance: objectives and design of the JEM-EUSO mission, Astrophys. Space Sci. Trans. 7, 477-482, 2011

[5] Kawasaki, Y. et al.: The focal surface of the JEM-EUSO instrument, Astrophys. Space Sci. Trans. 7, 167-169, 2011

[6] Ebisuzaki, T., et al.: The JEM-EUSO Project: Observing Extremely High Energy Cosmic Rays and Neutrinos from the International Space Station, Nuclear Physics B-Proceedings Supplements, 175-176, 237-240, proceedings of the XIV International Symposium on Very High Energy Cosmic Ray Interactions, 2008

[7] Kajino, F.: The JEM-EUSO mission to explore the extreme Universe, Nuclear Instruments and Methods in Physics Research Section A: Accelerators, Spectrometers, Detectors and Associated Equipment 623, 422-424, 1st International Conference on Technology and Instrumentation in Particle Physics, 2010

[8] Takahashi, Y. and the JEM-EUSO Collaboration: The JEM-EUSO mission, New Journal of Physics 11, 065 009-+, 2009 